\title{
Búsqueda Fenotípica del Retardo Mental Ligado al Sexo: Síndrome del $\mathrm{X}_{\mathbf{q}}$-Frágil
}

\author{
Ives Lacassie $S .{ }^{(1)}$. Alejandro Zavala $\mathrm{B},{ }^{(2)}$, Bianca Curotto L. (3), M. Angélica Alliende R. (3), \\ Isidora De Andraca $0 .{ }^{(4)}$
}

\section{Phenotypic Screening for X-Linked Mental Retardation: Fragile $\mathrm{Xq}_{\mathrm{q}}$ Syndrome}

\begin{abstract}
Current knowledge about X-linked Mental Retardation (MR) associated to Marker X chromosome (Xqfra) is reviewed. This is one of the most common causes of MR, and, probably, the first chromosomal alteration related to biochemical abnormalities susceptible to treatment.

We report the results of a screening for Fragile $X$ syndrome by testicular size moasurement and other phenotypic characteristics, on 85 institutionalized mentally retarded males. Preliminary results show an incidence of macroorchidism (MO) of $17.6 \%$ Considering only the population older than 15 years of age, the incidence raised to $28.6 \%$ (vs. $6.9 \%$ in prepuberal boys). According to other unspecific phenotypic characteristics, $18.8 \%$ males could be supected of having this syndrome. The incidence of $\mathrm{MO}$ in relation to the diagnostic categories of this unselected mentally retarded group is discussed. As it has been suggested that testicular size alone may be a useful screen to select patients with the fragile $X$ syndrome, and although the $\mathrm{Xq}$ fra is still to be demonstrated, these preliminary results suggest the existence of this Syndrome in our Country.
\end{abstract}

El Retraso Mental (RM) no específico ligado al sexo (X-1) constituye una causa muy frecuente de RM. De todas las afecciones genéticas, el RM asociado a fragilidad del cromosoma $X$ (Xqfra) ha sido, aparentemente, la que ha concentrado el mayor interés y número de publicaciones, en los últimos 3 años.

A fines de 1981 se presentó una revisión del tema (1) destacando los puntos de mayot interés para los pediatras. Algunos de los hitos históricos presentados se señalan en la Tabla 1. Los fenotipos de los 3 sindromes mejor delineados de RM ligados al cromosoma $X$ se presentan en la Tabla 2. Las características clinicas đel RM-X-I asociado a Xqfra se indican en la Tabla 3.

Desde fines de 1981 hasta mayo de 1982, se han descrito nuevos avances. Algunos de éstos se incluyen en la Tabla 4. Su importancia en Pediatría ha sido enfatizada (2-4).

1 Jefe Unidad de Gerética, INTA, Universidad de Chile.

2 Estudiante de Medicina, Fac. de Medicina Norte, Universidad de Chile.

3 Tecnólogo Médico, Unidad de Genética, INTA, Unjversidad de Chile.

4 Psicóloga, Servicio de Psiquiatría Hospital Sótero del Río.
Interesados en la identificación y posterior evaluación del tratamiento de este síndrome en nuestro medio, se realizó el tamizaje fenotípico de una población de sujetos con RM institucionaliza. dos.

\section{MATERIAL Y METODO}

Se estudiaron 85 sujetos de sexo masculino, internados en el Pequeño Cottolengo (Santiago, Chile), durante los meses de febrero y mario de 1982. A cada sujeto se le llenó una ficha ad hoc. La información requerida fue obtenida de los registros de la institución, de las historias clínicas hospitalarias, $y$ de estudios previos realizados en esa institución. El examen físico fue dirigido, considerando fundamentalmente los aspectos descritos en este síndrome (Tabla 3). Especial cuidado se tuvo en el examen y cuantificación de los genitales. El volumen testicular fue evaluado utilizando la fómula de Hansen (V.T. $=\pi / 6 \times \mathrm{L} \mathrm{X}$ $\left.A^{2}\right)(5,6)$, y su distribución en percentiles fue calculada de acuerdo a las tablas de Zachmann et al (7). El pene fue evaluado según las tablas de Schonfeld, y las características sexuales secundarias de acuerdo a los estadios de Tanner (8).

En este estudio se consideró macro orquidismo un volumen testicular sobre 2 desviaciones standard 
Tabla 1

Algunos Hitos Históricos

1932 WAARDENBURG: Sugiere algunas formas de RM de causa cromosómica.

1936-38 PENROSE: En 1.280 casos encuentra exceso de hombres con RM (25\%)

1943.65 MARTIN y BELL, ALLAN et al., RENPENNING. Describen RM X-I.

1965 REED: Matrimonio \& RMX $\& \mathrm{~N}=9,1 \% \mathrm{RM}$. Matrimonio of N X $₹$ RM: $19.4 \%$ DEKABAN: Reporta el primer sitio frágil en brazo largo de un $\mathrm{C}$.

1967-69 LUBS: Nota asociación de marcador del cromosoma X y RM-XL. Planteando posibilidades de diagnóstico prenatal.

1968 LEJEUNE: Muestra por primera vez que los sitios frágiles eran heredables.

1969 HECHT: Acuña el término "sitio frágil".

1968-74 LEHRKF: Postula que 25-50\% de todos los RM se deben a genes ligados al sexo.

1969.71 ESCALANTE, GRUNSPUN y FROTA PES. SOA: Describen RM X-L más macrogenitosomía.

1971-74 TURNER: En 2.345 escolaies con $\mathrm{Cl}$ : $35-55$ encuentra exceso de parejas de hermanos sobre hermanas $(32 \%+$ de $\phi$ que $d)$ y calcula: - Frecuencia portadoras: $0,74 / 1000$.

- Frecuencia hemicigotos: 0,53/1000.

- $10-18 \%$ de $\delta^{*}$ con RM sin diagnóstico establecido tendrían el S. de Martín-Bell o Renpenning (RM sin estigmas físicos).

1975-78 TURNER, CANTU, BIEDEMAN, BOWEN $y$ otros: Confirman la asociación RM X-L y macro-orqudismo.

1976-77 WIENER, GIRAUD, HARVEY y otros: Confirman la asociación de RM X-L y marcador cromosoma $X$.

1977-79 SUTHERLAND: Relaciona los sitios frágiles heredables. (Cr. X y autosomas) con características del medio de cultivo ( $\uparrow$ Metotrexato, $\downarrow$ formil-tetrahidrofolato).

1978 TURNER et al.: Nota que los RM con marca-
TURNER: Demuestra la expresión en portadoras $-7 \%$ de $\%$ fenotípicamente normales con RM moderado presentan el X Fra.

TURNER: Aproximadamente 1/3 de las familias con RM ligado al sexo tienen el Síndrome X del sitio frágil. Su expresión en portadoras contribuye al RM maderado en niñas.

Numerosos investigadores contribuyen a la delineación del Síndrone.

LEJEUNE: Por primera vez efectúa el tratamiento parenteral de un paciente con RM y X-Fra, con 5 formil-tetrahidrofolato (50 MG JM/Día $\times 8$ días), describiendo disminución de las caracteristicas psicóticas con desaparición concomitante de la laguna (X-Fra.) Realiza el tratamiento de 18 pacientes demostrando mejoría del Síndrome psicótico.

dor $\mathbf{X}$ tenian macro-orquidismo.

1979 LEJEUNE: Propone su teoría que en el RM está alterado el metabolismo de los monocarboros.

1980 LEJEUNE: Describe que la L-serina, 5 amino levulinato y L-4 hidroxiprolina, reducen la frecuencia de anomalias cromosomáticas in vitro sugiriendo que el sitio frágil depende del meta. bolismo de los monocarbonos.

HOWARD-PEEBLES et al.: Demucstran la dependencia de metionina para la expresión del FRA (X) (q 28)

MARTIN et al:: Demuestra la inactivación al azar de los $\mathrm{X}$ en las portadoras.

HERBST: Describe una prevalencia de portadoras de 2.4/1000 y de hemicigotos. 1.8/1000. HERBST y Miller: Calculan existirían 7-19 genes ligados al sexo que pueden causar RM no específico.

RM-X-L: Causaria 25\%de los RM en hombres y sería más frecuente que el $S$. de Down.

JACKY y DILL: Logran la expresión del Fro $X$ q 28 en fibroblastos.

Tabla 2

Fenotipo sindromes con Retaudo Mental

ligado al Cromosoma $\mathrm{X}$

1944: Allan, Herndon y Dudley

1962: Renpenning
Hipotonía

Atrofia y Debilidad

Muscular

Retraso Psicomotor

Alt. Lenguaje

(Probl. Verbal)

Microcefalia

Talla Baja
Cont.

1962: Renpenning (Manifestaciones en

Hcterocigotas:

Excepcional)

1969: Escalantc, Grunspun ： Retraso Mental y Frota Pessoa
Macrogenitosomia (Pubertad) 
Fenotipo RM Ligado al Sexo (X Q FRA)

\section{a) RM Moderado a Profundo}

b) Ctecimiento dentro de límites normales: (Talla normal, PN y CC Normales o Aumentados).

c) Facie: Frente prommisentc

Base Nasal Ancha

Hipoplasia Relativa del Piso Medio

Prognatismo

Paladar Ojival

Orejas Grandes y Prominentes

(Iris celeste - Tambićn letetocigotas) d) Genitales: Macro-Orquidismo (Bilat. o Unilateral).

e) Alteraciones Neurológicas: Convulsiones

Espasticidad y Escoliosis

f) Deficits Verbal:

Retraso del lenguaje

Lenguaje repetitivo

g) Problemas Conductuales: (Conducta Psicótica)
Hiperactividad

Ansiedad

Cambjos de carácter

Autismo

Tabla 4

Algunos Trabajos Recientes

DAKER et al. (Lancet 1: 780, 1981): Describe el X frigil en 2 hermanos de inteligencia normal.

PASSARGE (Clin. Genet. 20: 391A, 1981): Sugiere al igual que Howard-Peebles, qua habría dos tipos de heterocigotas: 1 sin RM y disminución acl $\mathrm{Xq}$ fra $\mathrm{y}$ 2. Con RM Y persistencia, independiente de la edad.

GLOVER (Am. J, Hum. Genet. 33: 234, 1981): Г'L sitio frágil (Xq $\mathrm{fra}$ ) se expresa por depleción de la dcoxitimidina monofosfato disponible para la síntesis de DNA. El uso do FUdR favorece la expresión del X fra.

MARENI y MIGEON (Am. J. HUM. Genet. 33: 252, 1981) y CARROLL y HOWARD-PEEBLES (Am. J. Hum. Genet. 33:826, 1981); Demuestran que el sitio frágil Xq 27 ó 28 , no tiene efecto sobre la expresion de genes localizados en esa zona (G6PD y HPRT).

GERALD (Pediatrics 68: 594,1981 ): Revisa cl RM X-L y $S$ Xq fra, enfatizando que todos los médicos, cspecialmente los pediatras deberian conocer este síndrome relativamente frecuente.

BROWN et al. (Lancet 2: 1055, 1981): Demuestra que la medición testicular puede ser un medio útil para seleccionar personas con RM con el Xq-fra.

WEBB et al. (Lancet 2: 1231, 1981): Confirman la transmisión a través de un hombre aparentemente normal $y$ sugieren la necesidad de incluirlos en estudios familiares.

JENKINS et al. (Lancet 2: 1292, 1981): Observan el $\mathrm{X}$-fra en cultivos retrospectivos de células amnióticas (usando 5 FLdr), demostrando la factibilidad del diagnóstico prenatal. Además demucstran que con este método, es posible obtener el $\mathrm{Xq}$ fra en fibroblastos de portadoras obligadas en que no se indujo en linfocitos.

HECHT at al, (Pediatrics 69: 221, 1982): Resume y actualiza el concepto de sicio frágil, tanto de autosomas como del cromosoma $X$, $y$ señala algunos preceptos prácticos para pediatras como: 1. Sospechar el X fra en todas las familias cor $R M$ ligado al sexo. 2. Sospechar el $X$ fra en todo hombre con RDPM o testículos aumentados. 3. Sospechar el $\mathrm{X}$ fra en toda mujer con retraso o problemas de aprendizaje. 4. Repetir estudios cromosómicos hecnos sin técnica especial para el
$X$ fra. S. Solicitar estudios cromosómicos buscando el $X$ fra.

SHAPIRO et al. (Lancet 1: 99, 1982).: Realizan el diagnóstico prenatal, prospectivo, de un feto afectado. Este fue confirmado en cultivo de sangre fetal obtenida por fetoscopia.

SUTHIRLAND y JACKY (Lancet 1: 100, 1982): Advierten de algunas dificultades y problemas ligados al diagnóstico prenatal.

HECHT ef al. (Am. J. M. Genet. 11:489, 1982): Actuajizan los métodos para obtener fragilidad en los cromosomas, especialmente el $X$.

BROWN et al. (Lancet 1: 100, 1982): Señalan la posible asociación del $\mathrm{S}$. del Xq fra con autismo, sugiriendo que se investiguc el $\mathrm{X}_{\mathrm{q}}$ fra en todo hombre con autismo de causa no establecida.

GARDNER et al. (Lancet 1 : 101, 1982): Señalan algunos aspectos y modificaciones técnicas que permiten la demostración consistente del X frágil en cultivos de fibroblastos (suplemento con L-metionina y uso de solución hipotónica de citrato de sodio).

LEJEUNE (Lancet 1: 273, 1982): Resume los fundamentos y resultados promisorios obtenidos en Ia terapia de sujetos con Xq fra, especialmente de los síntomas psicóticos. La posibilidad de tratamiento para esta afección debería poner énfasis en los aspectos curativos y no preventivos (diagnóstico prenatal y aborto) de csta afección.

UCHIDA y JOYCE. (Am. J. Hum. Genet. 34: 286, 1982): En portadoras heterocigotas demuestran que la expresión de la fragilidad y capacidad mental son secundarias a la inactivación del X normal (consistente con Hipátesis de Lyon).

RHOADS (Pedjatrics 69: 668, 1982): Puntualiza algunos aspectos de interćs del $\mathrm{S}$. Xq fra, señalando que este sindrome probablemente afecta todos los grupos étnicos, dependiendo su diagnóstico de los conocimientos del pediatra para sospechar exte síndrome y de las facilidades y técnicas citogenéticas existentes).

HARPEY (Pediatrics 69: 670, 1982): Enfatiza la posibilidad de tratamiento y prevención de RM en este Síndrome y describe los resultados satislactorios obtenidos en el tratamiento de 3 miembros de una familia. 
$(\geqslant 26.6 \mathrm{ml})$. El resto de las características fenotípicas fueron cuantificadas sólo en algunos casos.

La información obtenida permite un análisis de acuerdo a: 1) Presencia o no de macro orquidismo. Este requjere una separación por edades, dado que el aumento testicular es frecuente en sujetos postpuberales, siendo excepcional en prepuberales.

2) Categorias diagnósticas. Un porcentaje de los pacientes tenía diagnósticos establecidos, como $\mathrm{S}$. de Down, fenilquetonuria, etc. Otro, un diagnóstico presumiblemente genético, aunque no exactamente determinado $y$ un tercer grupo, sin diagnóstico o RM de causa desconocida. 3) Número de caracteristicas fenotipicas, fundamentalmente fa- ciales, en las que 4 o más serían sugestivas de este sindrome.

\section{RESULTADOS}

Los resultados obtenidos se presentan en la Tabla 5. La incidencia global de macro orquidismo obtenida es $17.6 \%$. De los 15 sujetos con MO, $3(20 \%)$ son prepuberales. La incidencia de MO en pre y postpuberales es 6.9 y $28.6 \%$, respectivamente.

La incidencia de MO de acuerdo a las 3 categorías diagnósticas sería $14.2 \%$ y $14.8 \%$ en las con diagnóstico establecido o sospechoso y $23.3 \%$ en el grupo de causa desconocida. La incidencia de "sospechosos", de acuerdo a las caracteristicas fenotípicas faciales seria $18.8 \%$.

Tabla 5.

Resultados tamizaje fenotípico para R. Y. X-1 (Xq -- fra)*

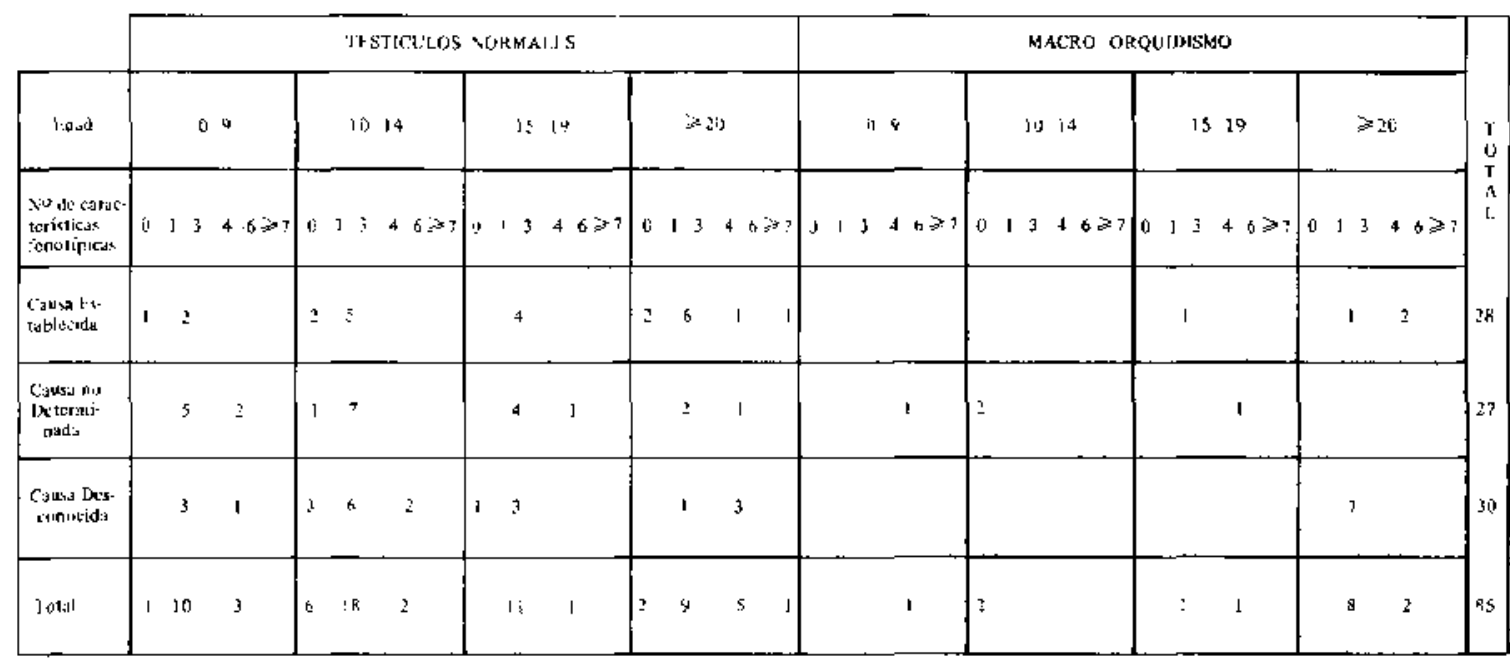

* No de casos encontrados según nomalidad o aumento testicular,

edad (en años). número de caractcrísticas fenotípicas y categoría diagnóstica.

\section{DISCUSION}

En los resultados obtenidos llama la atención el hallazgo de un $17.6 \%$ de $\mathrm{MO}$, que alcanza un $28.6 \%$ en adultos. Es de interés la presencia de MO en prepúberes, ya que aunque ha sido descrito en recién nacidos (5), es excepcional antes de la pubertad.

Si uno excluye los sujetos prepuberales, así como los con diagnósticos establecidos (donde había $5 \mathrm{~S}$. de Down, que no tienen MO...), la incidencia de $\mathrm{MO}$ alcanza un $45.8 \%$.

La mayor incidencia de MO en el grupo de RM de causa desconocida era esperable. La presencia de $\mathrm{MO}$ en sujetos con diagnóstico "supuestamen. te" establecido hace dudar de algunos de esos diagnósticos como "anoxia del parto", "encefalo- patía prenatal", etc., que no descartan que la causa real del RM sea el RM-X-1 con MO. Esto nos ha motivado a plantear la necesidad de un estudio detallado, clínico y citogenético, del $100 \%$ de la población institucionalizada. Esto permitirá el diagnóstico preciso que, en estos momentos, alcanza a un $33 \%$ con diagnósticos supuestamente establecidos, no etiológicos en su mayoría.

La incidencia de "sospechosos" de acuerdo a la presencia de características faciales liamativas, alcanzaría a un $18.8 \%$ Estas características no fueron y no siempre es posible cuantificarlas, por lo que no tienen el valor de la medición testicular que fue hecha rigurosa y objetivamente. La presencia simultánea de facie característica y $\mathrm{MO}$ alcanza un $7.1 \%$ en la población estudiada, corregida para la edad. La presencia de una y/u otra alcanzaría a un $31.7 \%$ 
Para establecer el diagnóstico de Síndrome del $\mathrm{Xq}$-fra es fundamental hacer el estudio cromosómico con medio pobre en ácido fólico. Brown y cols. en un estudio reciente y similar al nuestro (9) encontraron, entre 15 sujetos con RM inespecifico de causa desconocida, un $33 \%$ de MO. De los 5 sujetos con MO, 4 presentaban el Xq-fra en el estudio cromosómico, sugiriendo que el volumen testicular puede ser un indicador útil para seleccionar posibles pacientes con el Sindrome del X frágil.

Este trabajo, aunque es sólo una visión fenotípica, basada fundamentalmente en la presencia de MO, permite plantear que el RM-X-l acompanaado de MO constituye una causa frecuente de RM en nuestro medio, tal como ha sido establecido en trabajos en el extranjero.

\section{COMENTARIOS Y PERSPECTIVAS}

Este Síndrome es probablemente la causa más frecuente de RM. Sin embargo, quizás el aspecto más interesante de este sindrome lo constituya la posibilidad de que sea el primer ejemplo en la historia de la citogenética, en que una enfermedad asociada a alteración cromosómica a través de una alteración bioquimica parcialmente comprendida, sea susceptible de ser tratada (10).

Interesados en un estudio colaborativo destinado a evaluar el tratamiento de estos pacientes (11), realizamos una evaluación preliminar psicológica y citogenética en 4 de los 7 sujetos con RM de causa desconocida y $\mathrm{MO}$, ninguno de los cuales sería sospechoso del síndrome de acuerdo a las características faciales. En 2 de ellos fue posible efectuar también un estudio genealógico.

\section{Evaluación Psicológica:}

Se realizó una evaluación utilizando 3 instrumentos: entrevista clínica psicopatológica, W.A.I.S. y Escala de Madurez Social de Vineland.

Sólo 3 de los 4 pacientes fueron evaluados con el W.A.l.S. y entrevista clínica, ya que uno de ellos presentaba excesiva ansiedad al contacto interpersonal y ausencia de lenguaje hablado.

Los 3 pacientes evaluados con el W.A.I.S. presentan un retardo intelectual severo, que se expresa en puntajes de C.I. total entre $46-<41$. EI análisis de los puntajes obtenidos en las escalas verbal y de ejecución muestran que el primero es levemente superior, aunque la diferencia no es significativa. Los puntajes en las diferentes subpruebas muestran escasa dispersión.

A través de la escala de ejecución se pudo observar que los 3 pacientes presentan torpeza
El lenguaje muestra importantes alteraciones. Tres de los 4 pacientes muestran dificultades en la articulación, e intelegibilidad en 2 casos. También se observó ausencia de conceptos y dificultades en la sintaxis. En todos los casos, el lenguaje es concreto y se apoya en abundante mímica complementaria. Se observó, además, dificultades en el lenguaje receptivo; las instrucciones simples deben ser dadas en repetidas ocasiones para conseguir su comprensión y/o ejecución.

De acuerdo con los datos obtenidos en la entrevista clínica, sólo uno de los 3 pacientes estudiados presenta alteraciones psicopatológicas especiales, en este caso de tipo paranoídeo. Otro de los pacientes entrevistados presenta trastorno de conducta y tendencia al aislamiento. Conforme a la información entregada por los adultos responsables de los pacientes, el joven que no pudo ser entrevistado presentaria tambiên un trastorno de conducta y tendencia al aislamiento.

El desarrollo social, evaluado en los 4 pacientes a través de la Escala de Madurez Social de Vineland, muestra un severo retraso. Los rendimientos alcanzados corresponden a una edad social entre 3 años y 9 años 7 meses. El nivel social se correlaciona con el puntaje de $\mathrm{Cl}$. El sujeto con más alto $\mathrm{CI}$ obtuvo también la mayor edad social.

Los resultados en el test de WAIS y de Vineland están, sin duda alguna, fuertemente influidos por las condiciones de institucionalización en que viven los pacientes evaluados. Todos ellos han per. manecido largo tiempo (5-10 años) en esa instjtución. Es por esto que la interpretación de los resultados debe realizarse con bastante cuidado. sin olvidar las condiciones de vida de los sujetos.

\section{Evaluación y Cítogenética:}

En 3 de los 4 sujetos aparecieron sitios frágiles en un cromosoma C (Fig. 1), idénticos a los reportados en la literatura extranjera. Sin embargo, el bandeo $G$ realizado para confirmar si se trataba de un $\mathrm{X}$, demostró que eran cromosomas 6 . En el 40 sujeto sólo se produjeron alteraciones inespecificas de fragilidad cromosómica. Este estudio se realizó con la técnica de Sutherland (12), según modificaciones de Howard-Peebles (13). Sin embargo, parece importante realizar nuevos estudios con las técnicas recientemente descritas (Tabla 4), que permiten una mayor positividad, incluso en casos previamente negativos. En todo caso, hay sugerencia que la presencia de sitios frágiles autosómicos predispondría a RM (3). 

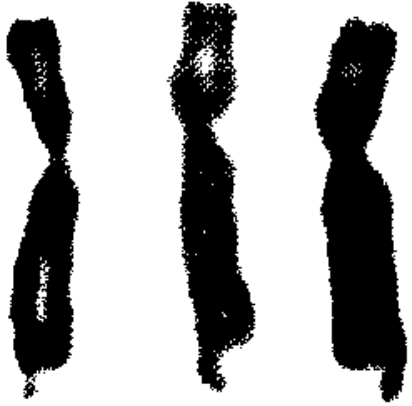

Figura 1.

Ejemplos de sitios frágiles en sujetos $1-3$

\section{Evaluación Genealógica:}

Dos de los 4 sujetos son hermanos (Fig. 2)* El cxamen de la hermana menor y de la madre reveló la presencia de $\mathbf{M}$ moderado en ambas, siendo compatible con herencia ligada al sexo con manifestaciones (RM leve a moderado) en un tercio de las portadoras (2-4). Aunque aún no se ha podido confeccionar la genealogía de otro de los sujetos, entre sus antecedentes figura la existencia de 3 hermanos con RM severo, aunque no institucionalizado, correspondiendo a un RM X-1. Del $4^{\circ}$ sujeto aún no tenemos antecedentes familiares.

La realización de estudios completos, que incluyan el análisis cromosómico con bandeos y búsqueda de sitios frágiles, en todo sujeto, hombre o mujer, con RM inespecífico leve a severo con o sin alteraciones fenotípicas, incluyendo $\mathrm{MO}$, es fundamental. No sólo sería importante para establecer la causa precisa, determinar las interrelaciones entre RM X-1, fragilidad cromosómica y caracteristicas fenotipicas, sino también para un consejo genético adecuado y establecer la posibilidad de tratamiento en un porcentaje importante de casos.

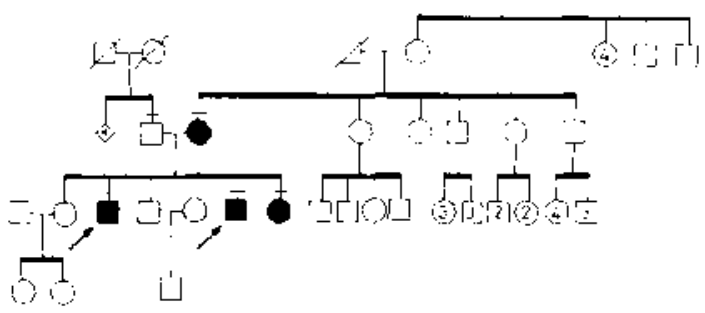

Figura 2.

Genealogía Sujetos 1 y 2*

\section{RESUMEN}

Se revisar los conocimientos actuales sobre el Sindrome de Retraso Mental (RM) ligado al sexo asociado a fragilidad del cromosoma $X$ (Xq-fra). Este constituye una de las causas más frecuentes de $R M, y$, probablemente, la primera alteración cromosómica relacionada a una alteración bioquimica susceptible de ser tratada.

Se presentan los resultados de una búsqueda de este sindrome en la población masculina de una institución para deficientes mentales, a través de la medición testicular y evaluación de otras caracte. rísticas fenotípicas. Resultados preliminares en 85 sujetos examinados revelan una incidencia de macro-orquidismo (MO) de $17.6 \%$. Al considerar sólo la población mayor de 15 años. la incidencia aumenta a $28.6 \%$ (vs. $6.9 \%$ en los prepuberales). De acuerdo a las otras caracteristicas fenotípicas, aunque menos específicas, un $18.8 \%$ sería sospechoso de presentar este sindrome. Se discute la incidencia de $\mathrm{MO}$ de acuerdo a las categorías diagnósticas en este grupo no seleccionado de retrasados mentales.

Si el tamaño testicular aislado es un buen medio dè selección de sujetos con el Sindrome del X frá. gil, como ha sido sugerido, aunque aún falta demostrar la fragilidad del cromosoma $X$, estos resultados preliminares sugieren la existencia de este Sindrome en nuestro medio.

\section{AGRADECIMIENTOS}

Agradecemos al Pequeño Cottolengo, especialmente a la Madre Fátima, por las facilidades dadas para este estudio. Al Dr. F. Novoa y al personal del Consultorio I y Hospital Barros Luco, así como a la Dra. M. Colombo, por proporcionamos las informaciones existentes sobre estos sujetos. A los Laboratorios Chile, Parke-Davis y Roche por proporcionarnos las drogas para la 2,a parte de este estudio y al Profesor Edison Cid, del Depto. de Tecnología Farmacéutica, Escuela de Química y Farmacia, por la confección de los comprimidos necesarios. Al Laboratorio Clinico Biotecnos por las facilidades para la realización de los exámenes citogenéticos. A la Sra. Viola Lyon por su paciencia y excelente labor secretarial.

\section{REFERENCIAS}

1 Lacassie, Y. : Retraso Mental Ligado al Sexo. Correlato Mcsa Redonda Atención lntegral del Niño Impedi-

- Estudios genéticos posteriores a la entrega del manuscrito. en uno de estos pacientes, demostraron la presencia de Fragilidad del Cromosoma $X$. 
do. IX Jornadas Nacjonales Sociedad Chilena de Pediatria. Lo Barnechea, 1981.

2 Gerald, P.S.: X-Linked Mental Retardation and the Fragile-X Syndrome. Pediatrics 68: 594, 1981.

${ }^{3}$ Hecht, F,; Glover, T.W.:; Kaiser-Hecht, B.; Fragile Sites on Chromosomes. Pediatrics 68: 121, 1982.

${ }^{4}$ Rhoads, F.A.: X-Linked Mental Retardation and Fragile-X or Marker-X Syndrome. Pediatrics 69: 668, 1982.

${ }^{5}$ Cantu, J.M.; Scaglia, H.E. Gonzólez-Diddi, M., Hernández-Jáuregui, P.; Morato, T.; Moreno, M.E.; Giner, J.; Alcántar, A.; Herrera, D.; Pérez-Poloctos, G.: [n. herited Congenital Normo-funcional Testicular Hyperplasia and Mental Deficiency. Hum. Genet. 41: 331 , 1978.

6 Prader, A.: El tamaño de los testículos: Valoración y significación clínica. Triángulo 7: 240, 1967.

${ }^{7}$ Zachmann, M.; Prader, A.; Kind, H.P.: Haffiger, H. Budliger, $\boldsymbol{H}$.: Testicular Volumen during Adolescence: Cross Sectional and Longitudinal Studies. Hely.
Paediat. Acta 29:61, 1974.

${ }^{3}$ Gardiner, L.I.: Endocrine and Genetic Diseases of Childhood and Adolescence. W.B. Saunders Co. Philadelphia. Second Edition, 1975.

${ }^{9}$ Brown, W.T.: Mezzcappa, P.M.; Jenkins, E.K.: Screening for Fragile $X$ Syndrome by Testicular Size Measurement. Lancet 2: 1055, 1981.

10 Lejeume, J,: Is the Fragile $X$ Syndrome Amenable to Treatment? Lancet 1:273, 1982.

11 Lejeune, J.: Project de recherche cooperatjue. V Congreso Latinoamericano de Genética, Viña del Mar, Chile, Octubre 1981.

12 Sutherland, G.R.: Heritable Fragile Sites on Human Chromasomes. I. Factors affecting expression in lymphocyte cultures. Am. J. Hum. Genet. 31:125, 1979.

13 Howard-Peebles, P.N.: Pryor, J.C.: Fragile Sites in Human Chromosomes. 1. The effect of methionine on the Xq fragile site. Clin. Genet, 19: 228, 1981. 